\title{
A Multifunctional Supramolecular Hydrogel for Prevention of Epidural Adhesion after Laminectomy
}

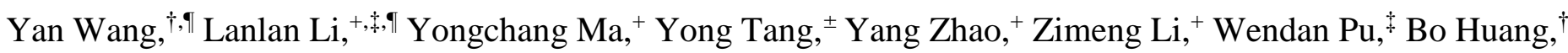
Xuan Wen, ${ }^{\dagger}$ Xiaojuan Cao, ${ }^{\dagger}$ Jiafei Chen, ${ }^{\|}$Wei Chen,, Yue Zhou, ${ }^{*}{ }^{\dagger}$ Jianxiang Zhang ${ }^{*,+, \perp}$

${ }^{\dagger}$ Department of Orthopaedic Surgery, Affiliated Xinqiao Hospital, Third Military Medical University (Army Medical University), Chongqing 400037, China

${ }^{+}$Department of Pharmaceutics, College of Pharmacy, Third Military Medical University (Army Medical University), Chongqing 400038, China

Department of Chemistry, College of Basic Medicine, Third Military Medical University (Army Medical University), Chongqing 400038, China

${ }^{ \pm}$Department of Orthopaedic Surgery, The 72 Hospital of Army, Huzhou 313000, China

"Department of Radiology, Southwest Hospital, Third Military Medical University (Army Medical University), Chongqing 400038, China

${ }^{\perp}$ Institute of Burn Research, State Key Laboratory of Trauma, Burn and Combined Injury, Southwest Hospital, Third Military Medical University (Army Medical University), Chongqing 400038, China

\section{Corresponding authors:}

Jianxiang Zhang, PhD, Prof.

E-mail: jxzhang1980@gmail.com,jxzhang@tmmu.edu.cn

Yue Zhou, PhD, Prof.

E-mail: happyzhou@vip.163.com 
P16-NP5

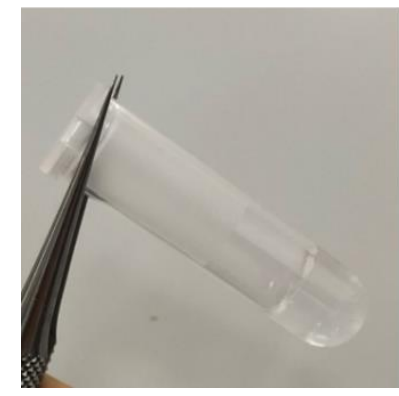

P18-NP5

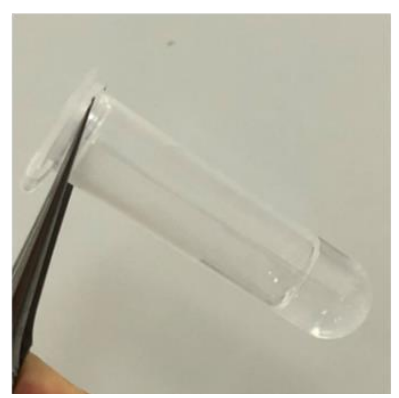

P20-NP5

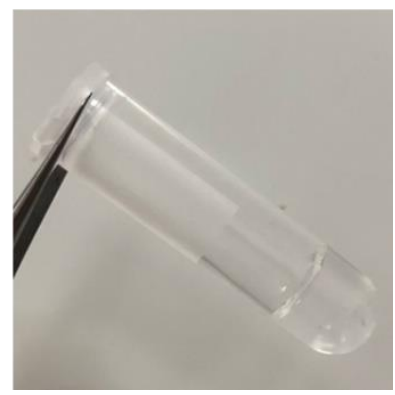

Figure S1. Digital photos of aqueous solutions containing varied concentrations of PX and TPCD NP at room temperature of $20^{\circ} \mathrm{C}$. For P16-NP5, P18-NP5, and P20-NP5, the concentration of TPCD NP was $5 \mathrm{mg} / \mathrm{mL}$, while the PX concentration was 16,18 , and $20 \mathrm{wt} \%$, respectively. 

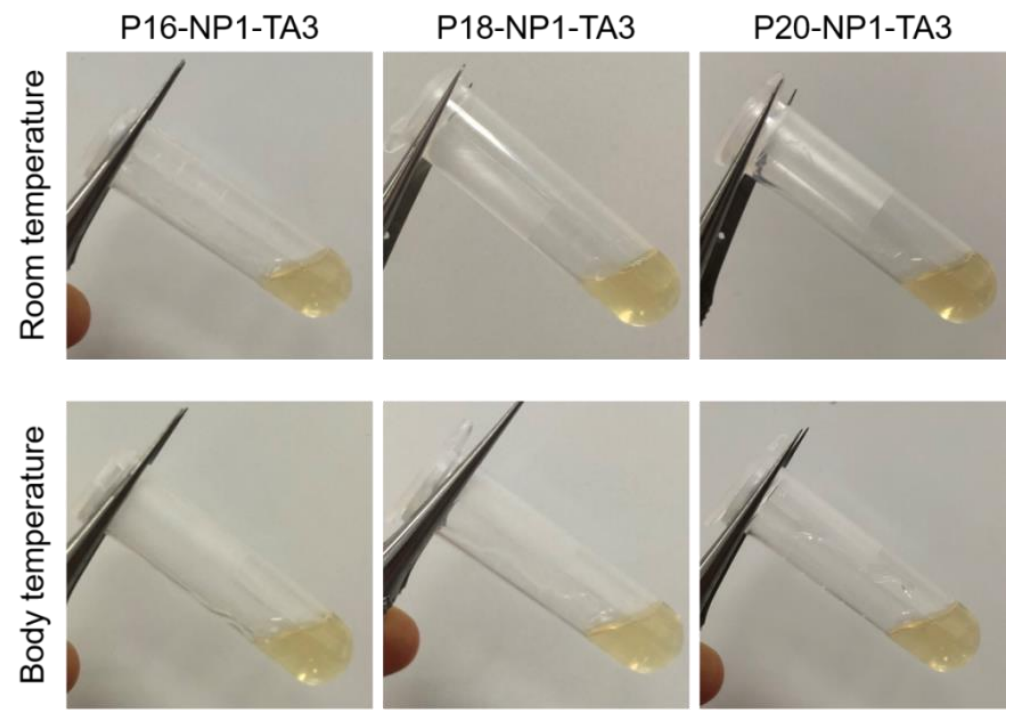

Figure S2. Digital photos of aqueous solutions containing various concentrations of PX, TPCD NP, and TA at room or body temperature. P16-NP1-TA3, P18-NP1-TA3, and P20-NP1-TA3 represent formulations containing $1 \mathrm{mg} / \mathrm{mL}$ TPCD NP and $3 \mathrm{wt} \%$ TA, with the PX concentration varied from 16, 18, to $20 \mathrm{wt} \%$, respectively. 
A

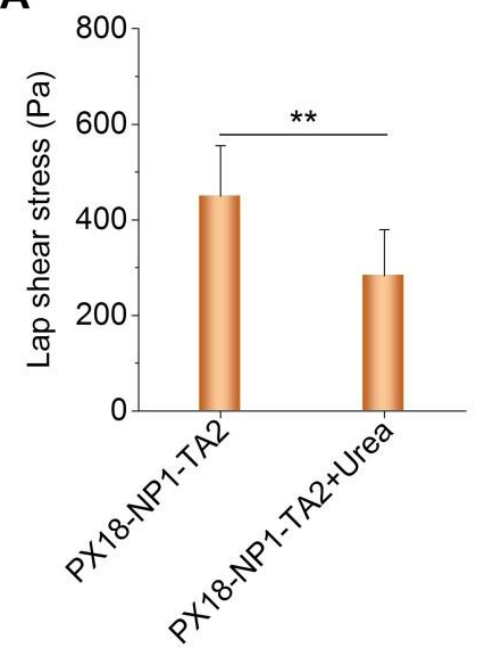

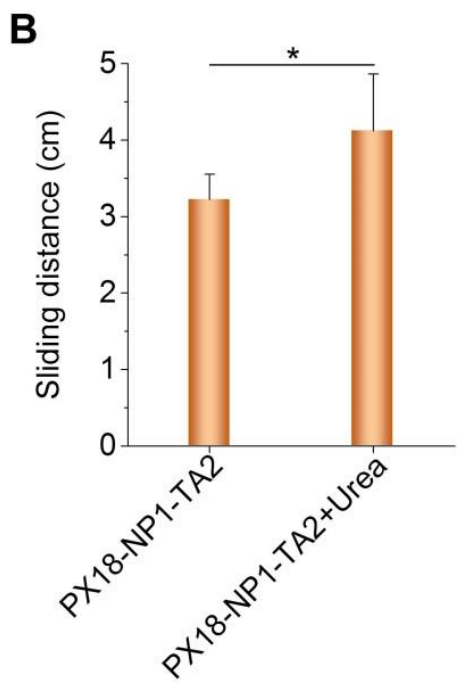

Figure S3. The effects of urea on bioadhesiveness of TA-containing hydrogels. (A) The quantified lap shear stress between rat muscle tissues bound together with PX18-NP1-TA2 hydrogels with or without 25\% urea. (B) Sliding distance for PX18-NP1-TA2 hydrogels with or without $25 \%$ urea. Data are presented as mean \pm SD $(n=$ 6). Statistical significance was assessed by one-way ANOVA tests. $* \mathrm{P}<0.05, * * \mathrm{P}<0.005$. 


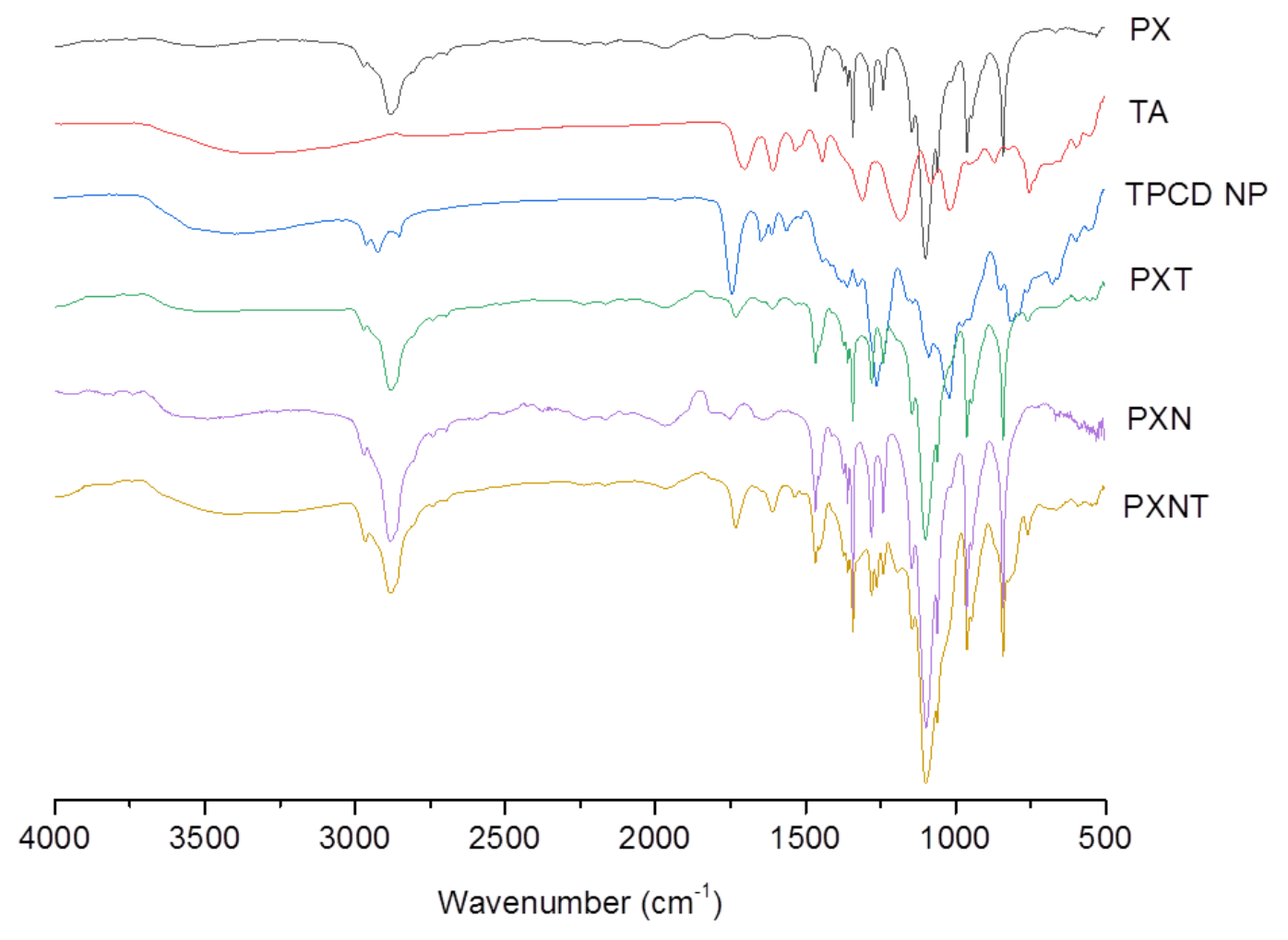

Figure S4. FT-IR spectra of various materials. PXT, a freeze-dried mixture of $18 \mathrm{wt} \% \mathrm{PX}$ and $2 \mathrm{wt} \% \mathrm{TA}$; PXN, a freeze-dried mixture of $18 \mathrm{wt} \% \mathrm{PX}$ and $1 \mathrm{mg} / \mathrm{mL}$ TPCD NP; PXNT, a freeze-dried mixture of $18 \mathrm{wt} \% \mathrm{PX}, 1$ $\mathrm{mg} / \mathrm{mL}$ TPCD NP, and $2 \mathrm{wt} \%$ TA. 

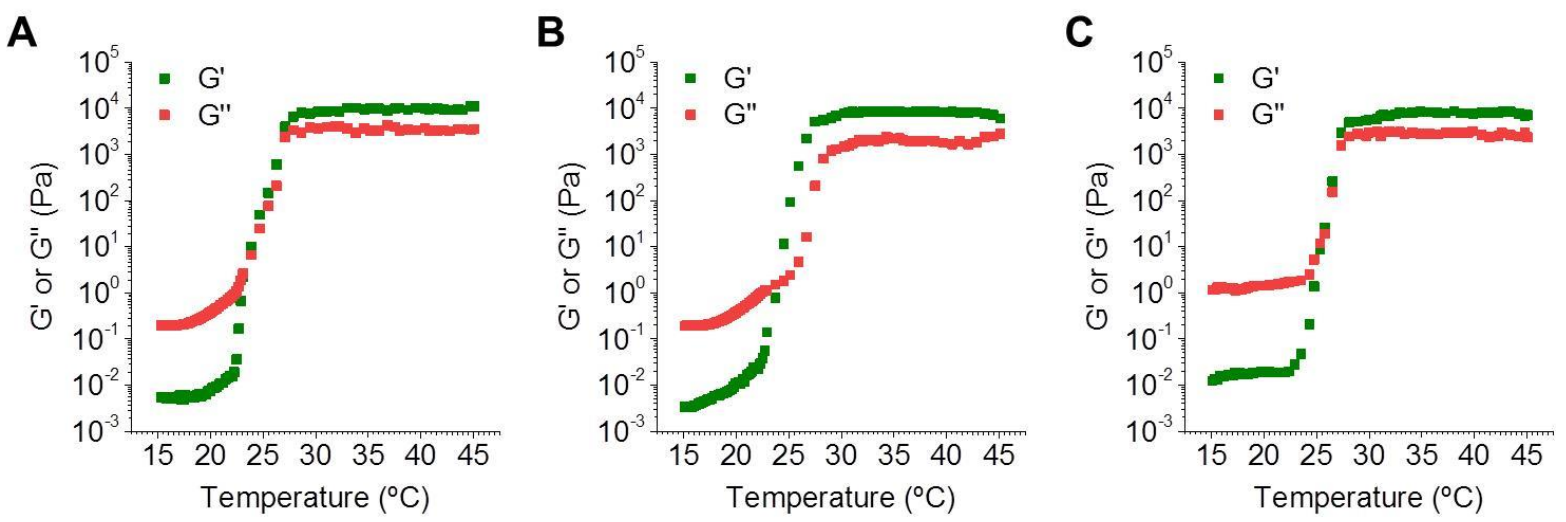

Figure S5. Temperature-dependent rheological behaviors of various formulations. (A) PX, $18 \mathrm{wt} \% \mathrm{PX}$. (B) PXN, a mixture of $18 \mathrm{wt} \%$ PX and $1 \mathrm{mg} / \mathrm{mL}$ TPCD NP. (C) PXT, a mixture of $18 \mathrm{wt} \%$ PX and $2 \mathrm{wt} \%$ TA. In all cases, $\omega=10 \operatorname{rad~s}^{-1}, \varepsilon=2 \%$. 


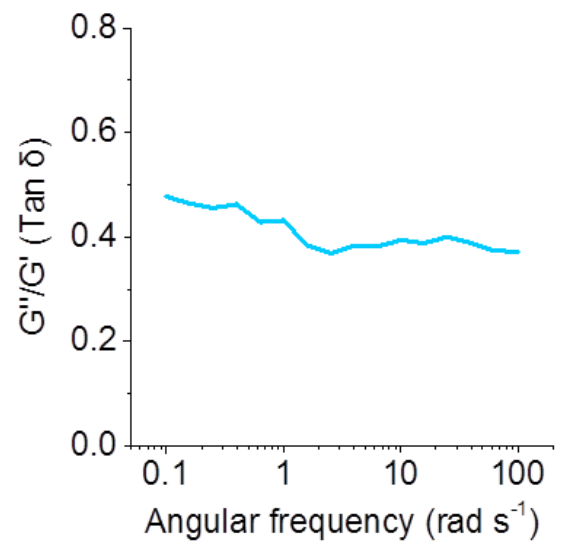

Figure S6. Angular frequency dependent changes in the values of $\mathrm{G}^{\prime \prime} / \mathrm{G}^{\prime}$ (i.e., Tan $\delta$ ). Data were calculated from $\mathrm{G}^{\prime}$ and $\mathrm{G}^{\prime \prime}$ data in Figure 3I. 
A

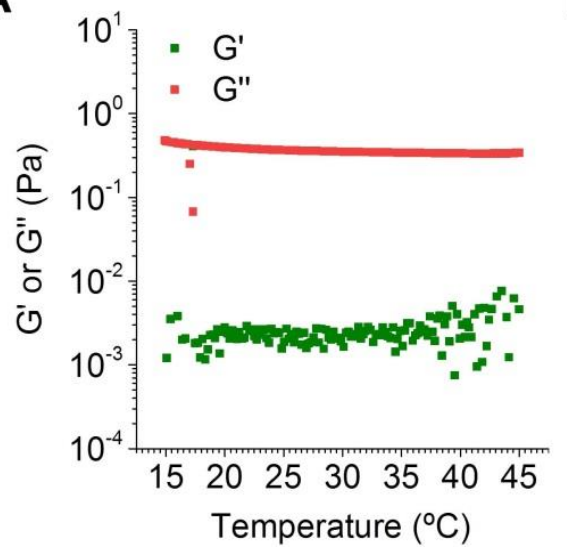

B

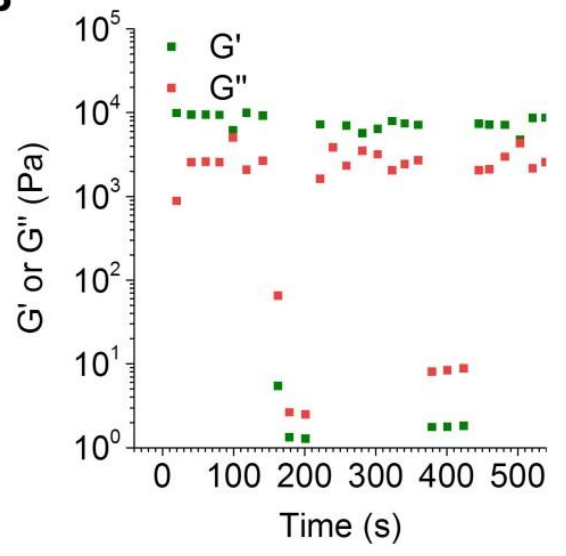

Figure S7. Effects of different additives on gelation and self-healing capacity of PXNT. (A) Temperaturedependent rheological behaviors of PXNT solution in the presence of $10 \mathrm{wt} \%$ sodium dodecyl sulfate $\left(\omega=10 \mathrm{rad} \mathrm{s}^{-1}, \varepsilon=2 \%\right)$. (B) Step-strain measurement of PXNT hydrogel in the presence of $25 \mathrm{wt} \% \mathrm{urea}$ $\left(\omega=10 \mathrm{rad} \mathrm{s}^{-1}, 37^{\circ} \mathrm{C}\right)$. The relatively high strain was $200 \%$, while the low strain was $2 \%$. 

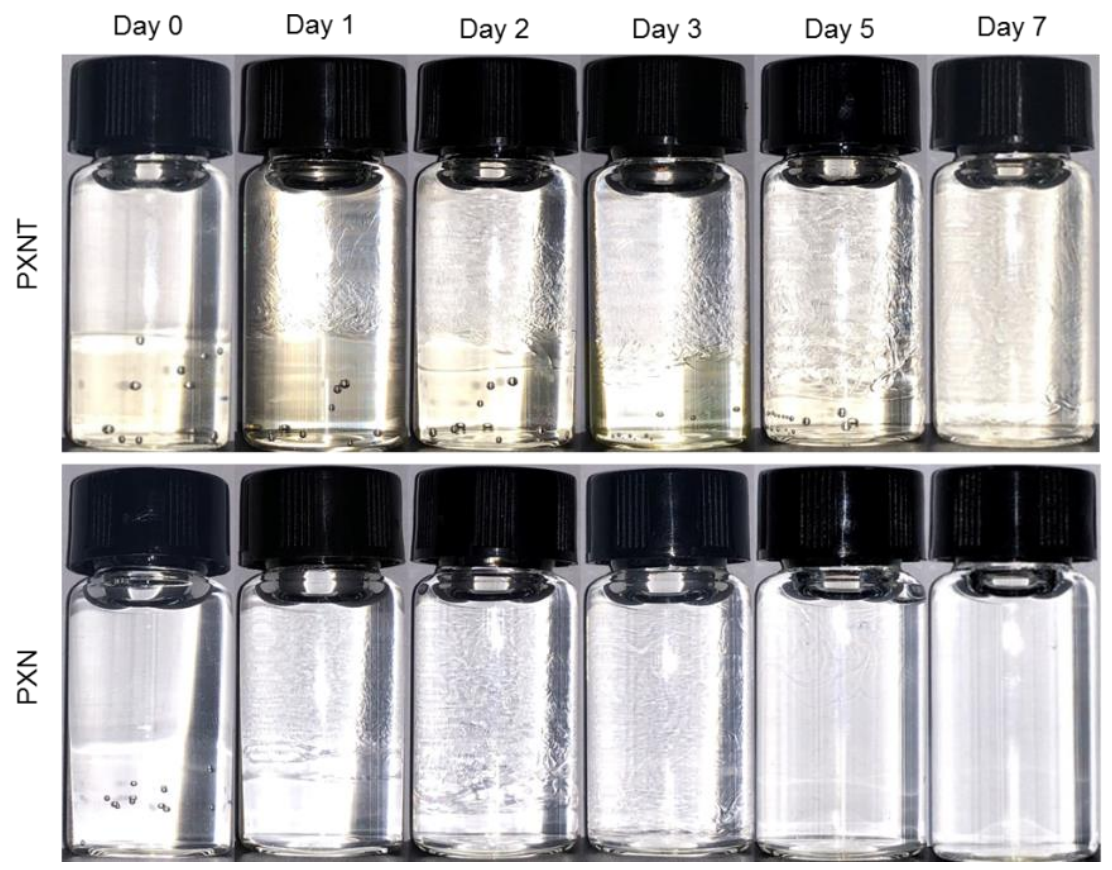

Figure S8. Digital photos showing erosion of hydrogels based on different formulations. Erosion tests were performed in $0.9 \%$ saline at $37^{\circ} \mathrm{C}$. PXNT, a mixture of $18 \mathrm{wt} \% \mathrm{PX}, 1 \mathrm{mg} / \mathrm{mL}$ TPCD NP, and $2 \mathrm{wt} \% \mathrm{TA}$; PXN, a mixture of $18 \mathrm{wt} \% \mathrm{PX}$ and $1 \mathrm{mg} / \mathrm{mL}$ TPCD NP. 


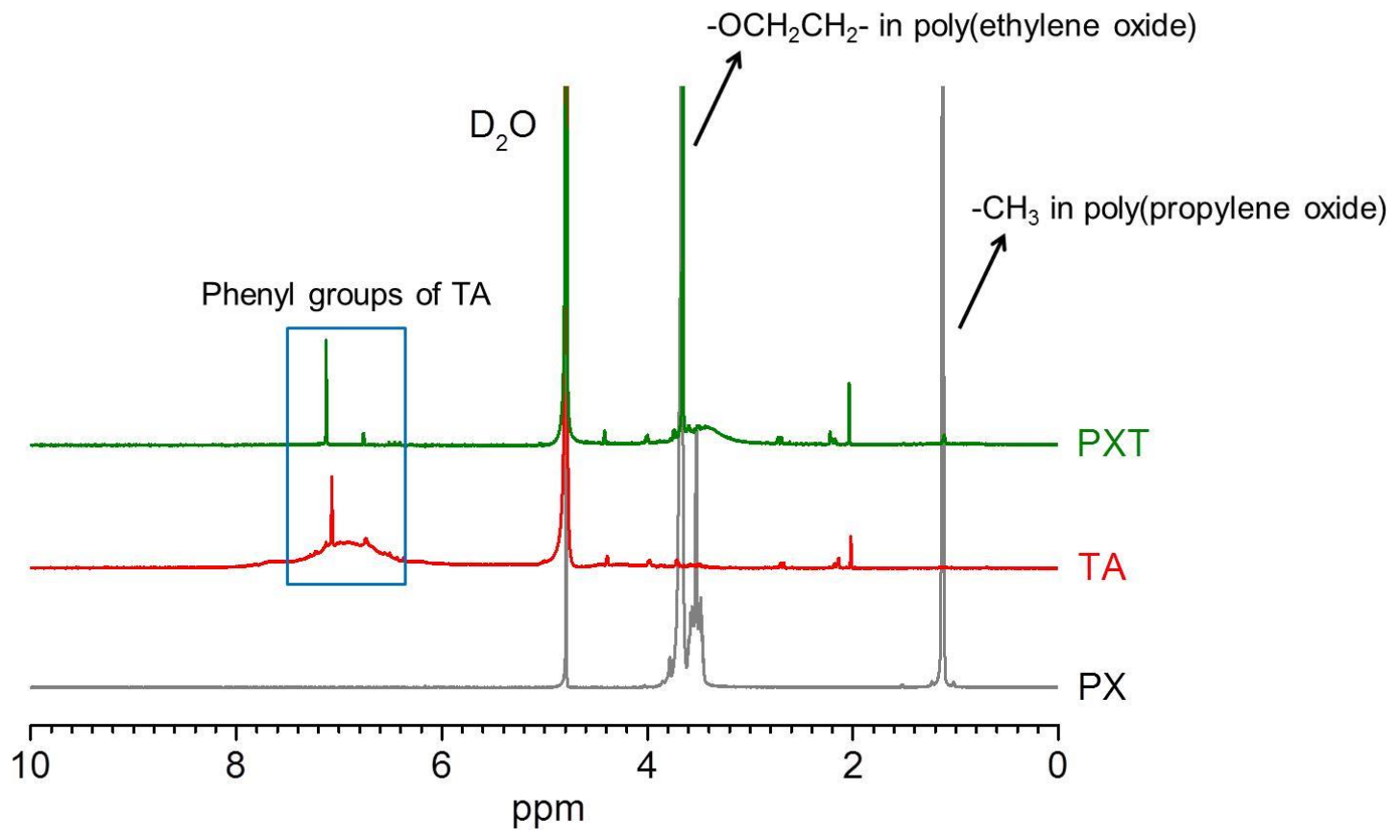

Figure S9. ${ }^{1} \mathrm{H}$ NMR spectra of different materials in $\mathrm{D}_{2} \mathrm{O}$. PXT is a mixture of TA and PX. 


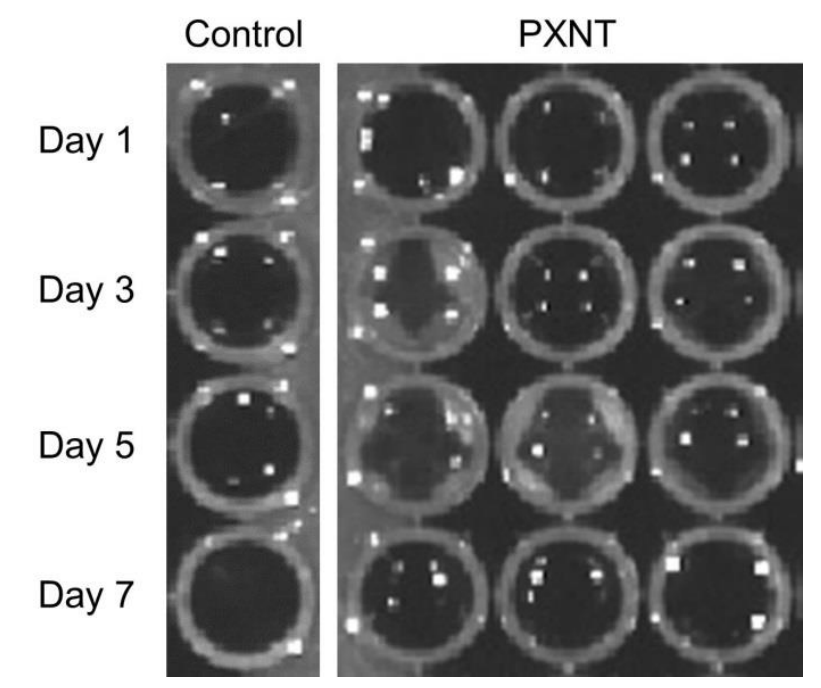

Figure S10. Representative ex vivo fluorescence images of blood samples at different time points after local injection in deep sacrospinalis of rats. 
A
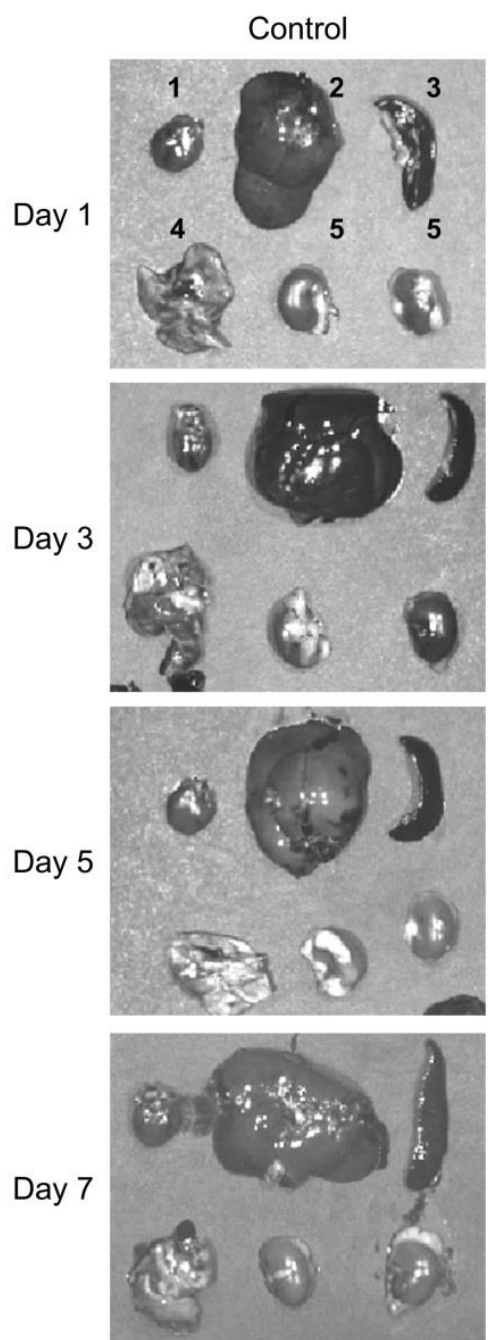

Radiant efficiency $\left(x 10^{7}, \mathrm{p} / \mathrm{s} / \mathrm{cm}^{2} / \mathrm{sr} / \mu \mathrm{W} / \mathrm{cm}^{2}\right)$
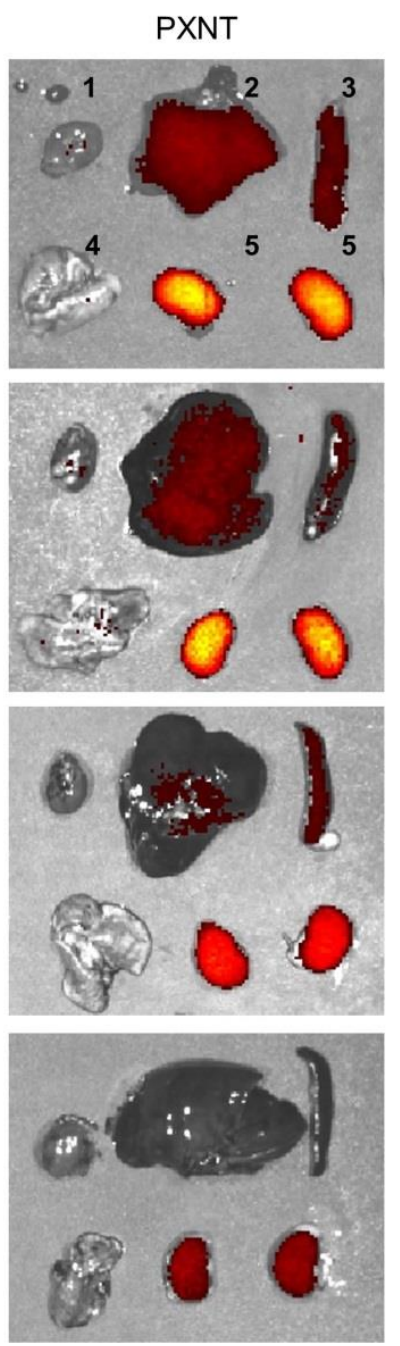

$\begin{array}{lll}3.0 & 4.0 & 5.0\end{array}$

B

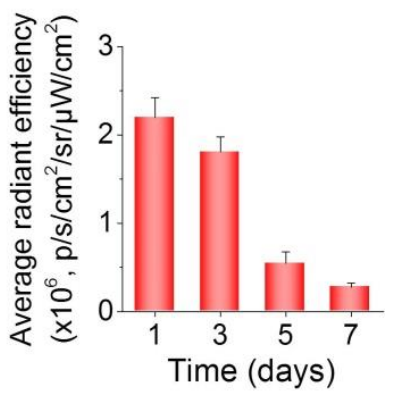

C

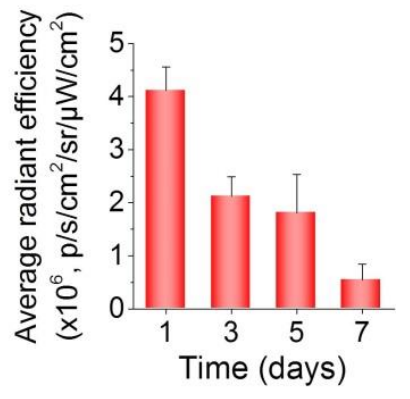

D

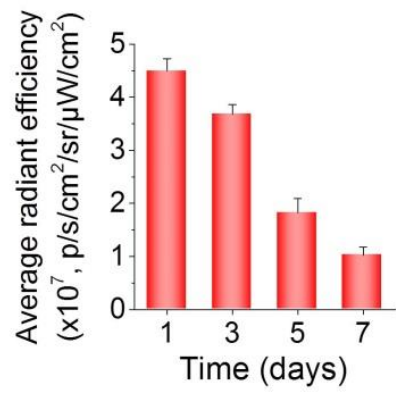

Figure S11. In vivo biodistribution of PXNT hydrogel in major organs after injection in deep sacrospinalis. (A) Representative ex vivo fluorescence images of heart, liver, spleen, lung, and kidneys at different time points after injection of Cy5-labeled PXNT in deep sacrospinalis of rats. (B-D) Quantitative analysis of fluorescence intensities of liver (B), spleen (C), and kidneys (D) at examined time points. Cy5-labeled PXNT was based on a mixture of 18 wt\% PX, 1 mg/mL Cy5-labeled TPCD NP, and 2 wt\% TA. 1, heart; 2, liver; 3, spleen; 4, lung; 5, kidneys. Data in $(B-D)$ are presented as mean $\pm S D(n=3)$. 
A

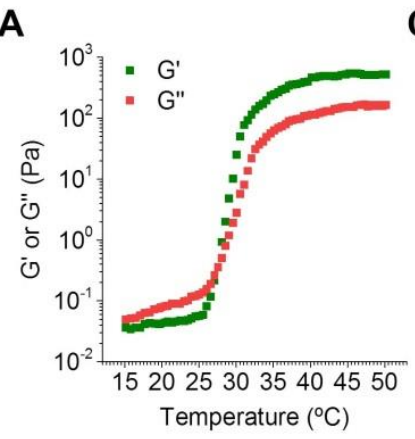

B

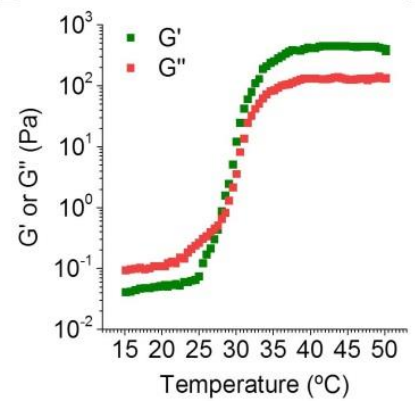

C

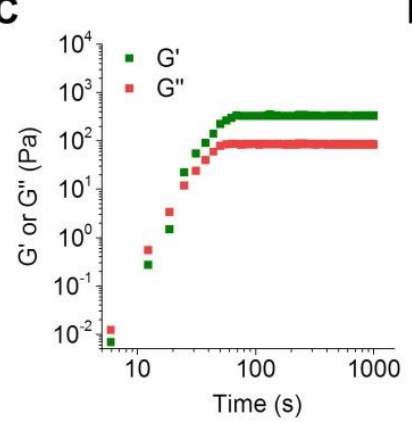

D

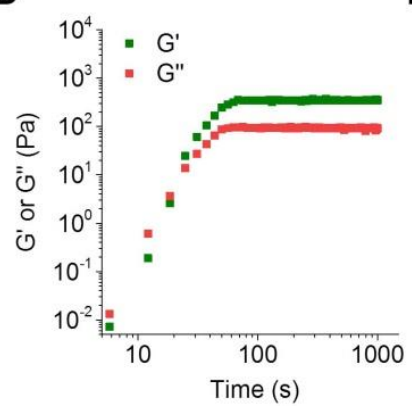

E

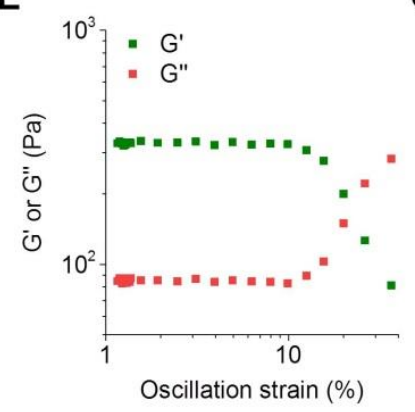

F

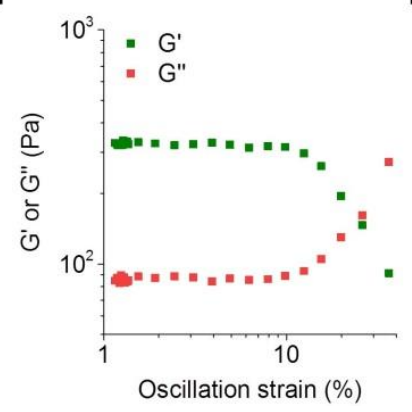

G

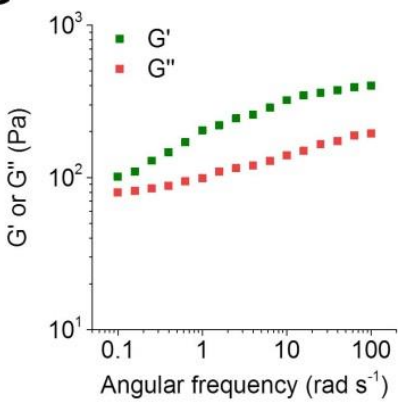

H

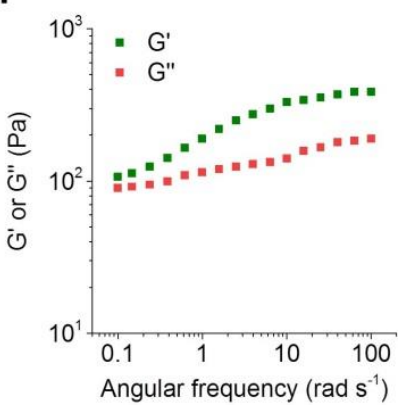

Figure S12. Rheological properties of aqueous solutions containing PL with or without TPCD NP. (A-B) Temperature-dependent rheological behaviors of $15 \mathrm{wt} \%$ PL solution (A) or PLN solution (B). In both cases, $\omega=10 \mathrm{rad} \mathrm{s}^{-1}, \varepsilon=2 \%$. (C-D) Time-dependent oscillatory rheology of $15 \mathrm{wt} \%$ PL hydrogel (C) or PLN hydrogel (D) at $37^{\circ} \mathrm{C}$. In all cases, $\omega=10 \mathrm{rad} \mathrm{s}^{-1}, \varepsilon=2 \%$. (E-F) Oscillatory strain-sweep tests of $15 \mathrm{wt} \%$ PL hydrogel (E) or PLN hydrogel (F) at $37^{\circ} \mathrm{C}$ and with $\omega=10 \mathrm{rad} \mathrm{s}^{-1}$. (G-H) Frequency-dependent rheology of $15 \mathrm{wt} \% \mathrm{PL}$ hydrogel $(\mathrm{G})$ or PLN hydrogel $(\mathrm{H})$ at $37^{\circ} \mathrm{C}$ and with $\varepsilon=2 \%$. In all cases, PLN denotes a mixture of $15 \mathrm{wt} \% \mathrm{PL}$ and $1 \mathrm{mg} / \mathrm{mL}$ TPCD NP. 
A

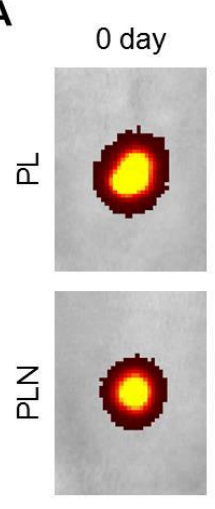

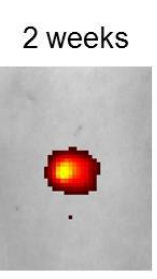

6
4 weeks

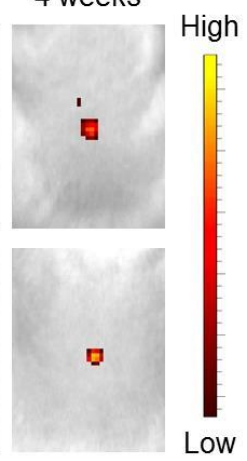

B

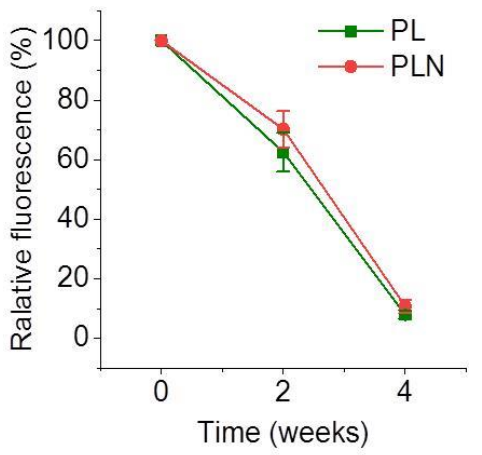

Figure S13. In vivo retention of PL-based hydrogels after intramuscular injection. (A) Representative fluorescence IVIS images of rats after intramuscular injection of aqueous solutions of Cy7.5-labeled PL (15 wt $\%$ ) or Cy7.5-labeled PL (15 wt\%) plus $1 \mathrm{mg} / \mathrm{mL}$ TPCD NP. (B) Quantitative analysis of fluorescence intensities at examined time points. PL, $15 \mathrm{wt} \%$ PL labeled with Cy7.5; PLN, a mixture of $15 \mathrm{wt} \%$ PL and 1 $\mathrm{mg} / \mathrm{mL}$ TPCD NP. Data are presented as mean $\pm \mathrm{SD}(\mathrm{n}=3)$. 


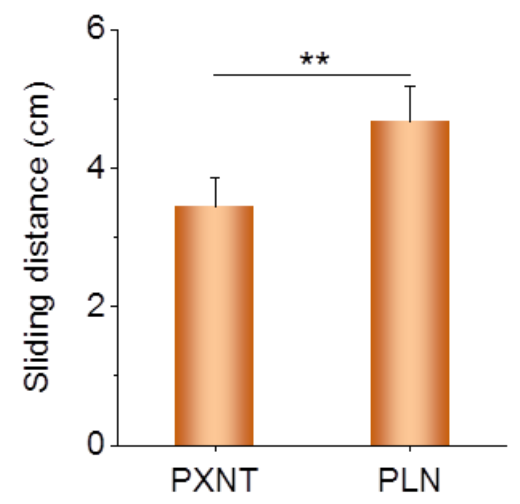

Figure S14. The quantified sliding distance for PXNT and PLN. Data are presented as mean \pm SD $(n=6)$. Statistical significance was assessed by one-way ANOVA tests. **P $<0.005$. 
A

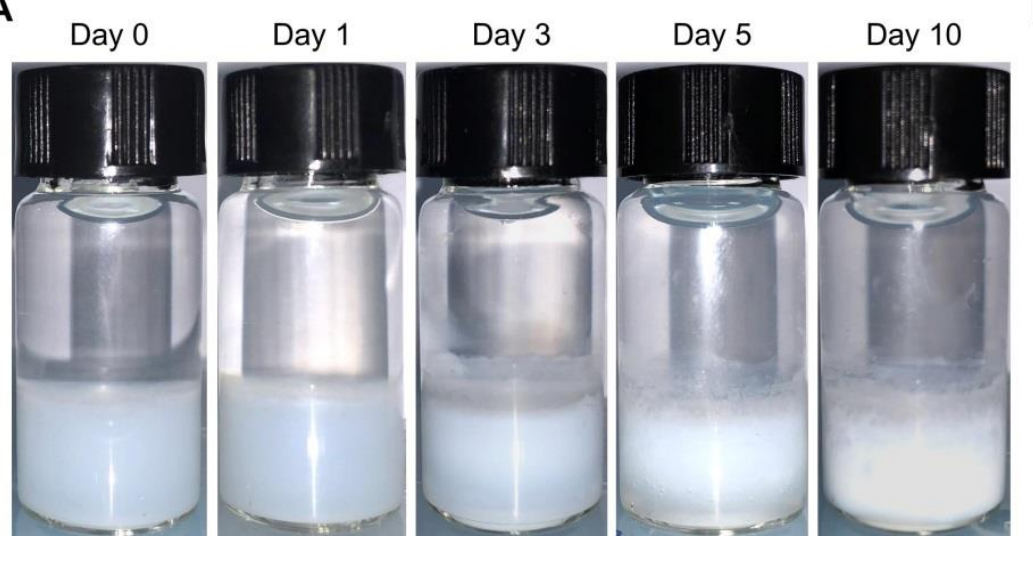

B

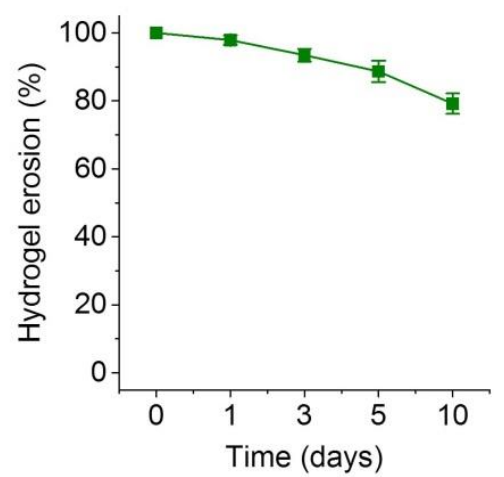

Figure S15. In vitro erosion of PLN hydrogel in $0.9 \%$ saline at $37^{\circ} \mathrm{C}$. (A) Representative digital photos of hydrogel samples at different time points during erosion tests. (B) The percentage of retained hydrogel weight after incubation for defined time periods. PLN, a mixture of $15 \mathrm{wt} \%$ PL and $1 \mathrm{mg} / \mathrm{mL}$ TPCD NP. Data are presented as mean $\pm \operatorname{SD}(n=3)$. 
A

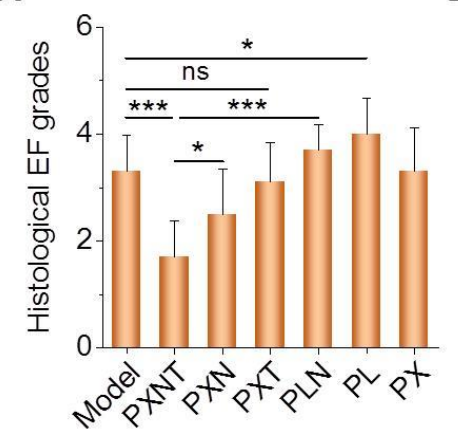

B

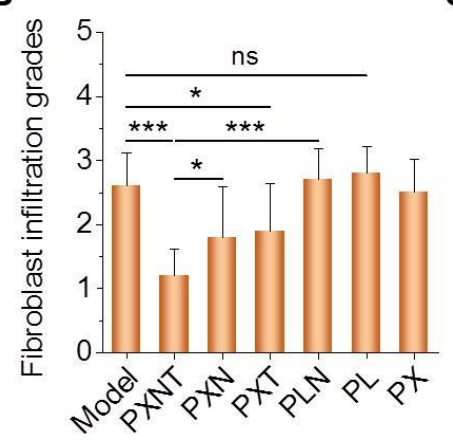

C

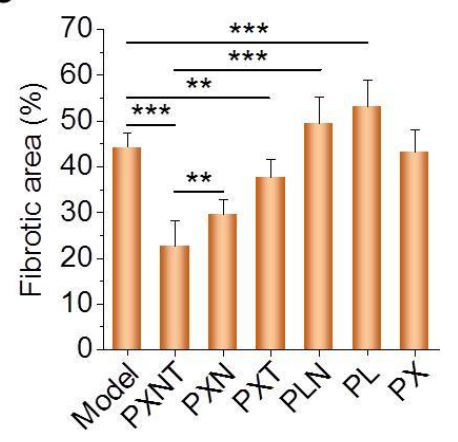

Figure S16. Semi-quantitative analysis of the EF degrees based on histological sections. (A) Quantitative analysis of EF grades. (B) Quantified fibroblast infiltration grades according to H\&E-stained sections at high resolution (400x). (C) Quantified percentages of fibrotic areas based on Masson-stained sections. Data are presented as mean $\pm \mathrm{SD}(\mathrm{n}=10)$. Statistical significance was assessed by one-way ANOVA tests. $* \mathrm{P}<0.05$, $* * \mathrm{P}<0.005, * * * \mathrm{P}<0.0005 ; \mathrm{ns}$, no significance $(\mathrm{P}>0.05)$. 


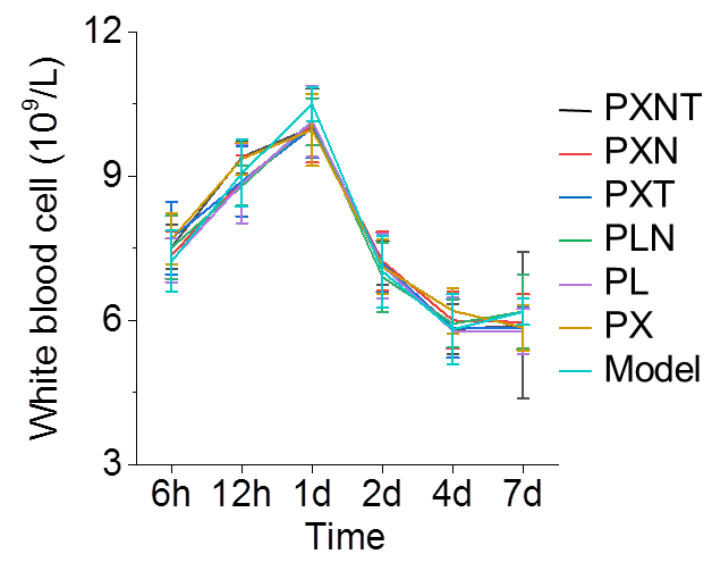

Figure S17. Changes in white blood cell counts at various time points after different treatments. Data are presented as mean $\pm \operatorname{SD}(n=12)$. 

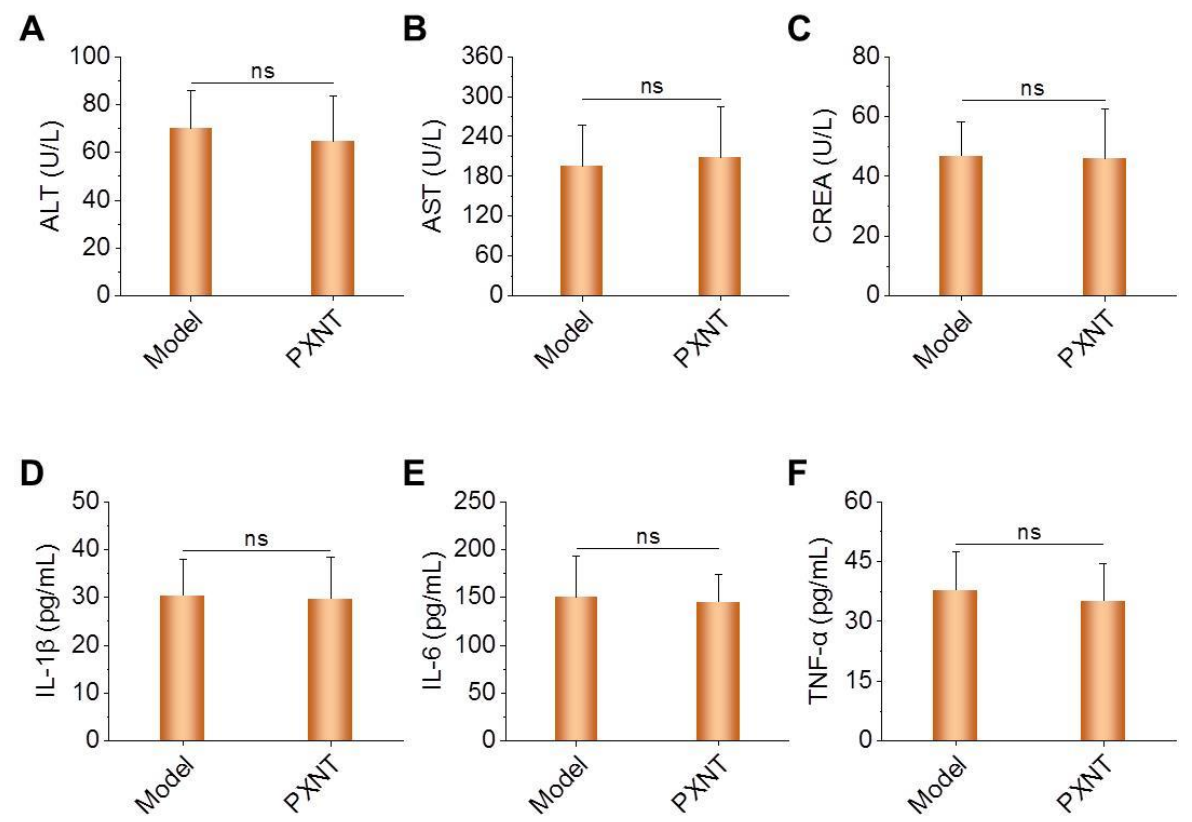

Figure S18. In vivo safety evaluations after treatment with PXNT hydrogel. (A-C) Typical biochemical markers related to hepatic and renal functions, including ALT (A), AST (B), and CREA (C). (D-F) The expression levels of IL-1 $\beta$ (D), IL-6 (E), and TNF- $\alpha$ (F) in blood serum from rats at week 4 after treatment with PXNT. Data are presented as mean $\pm \mathrm{SD}(\mathrm{n}=6)$. Statistical significance was assessed by one-way ANOVA tests. ns, no significance. 

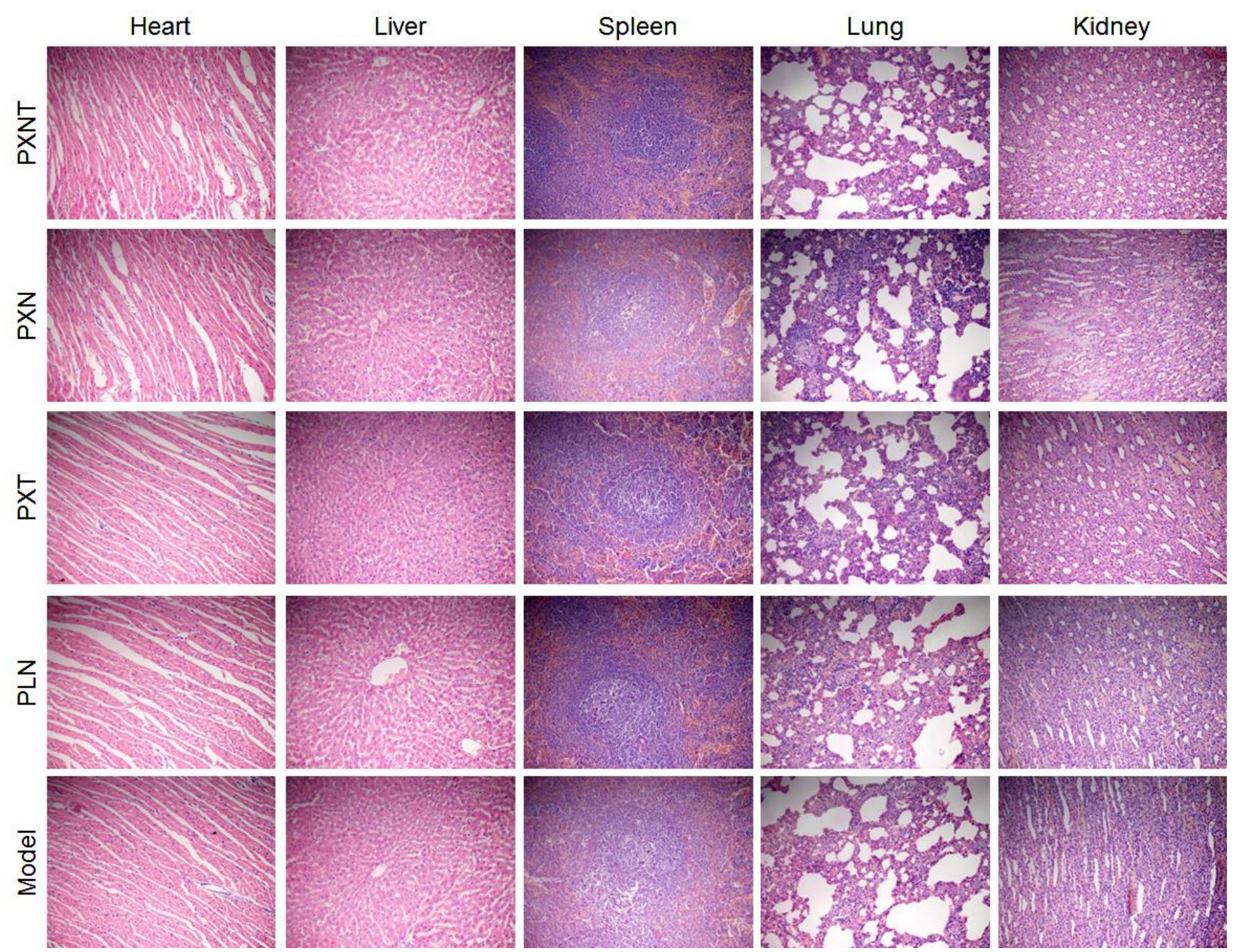

Figure S19. H\&E-stained histopathological sections of major organs resected from rats subjected to different treatments. 

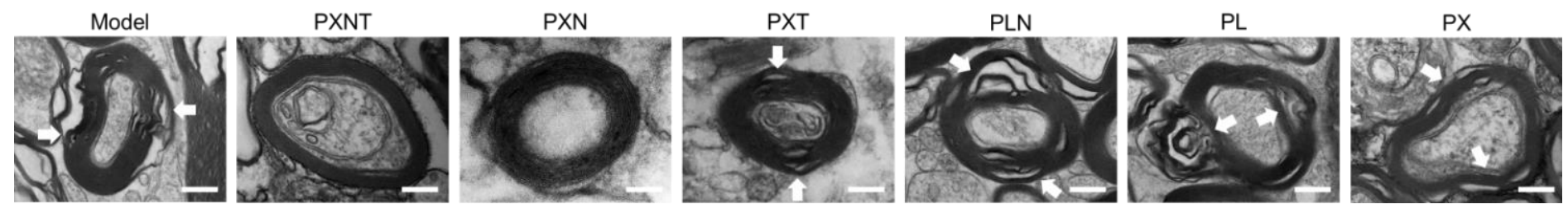

Figure S20. TEM images of spinal cord morphology at week 8 after treatment with various formulations. Neurodegeneration is characterized by the existence of abnormal myelin sheath (white arrow). Scale bars: $1 \mu \mathrm{m}$. 
A

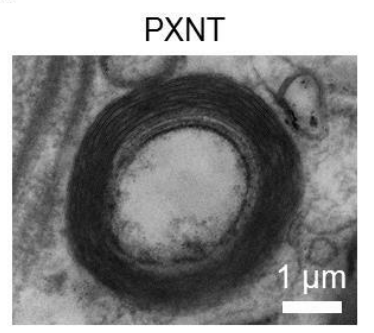

Interceed

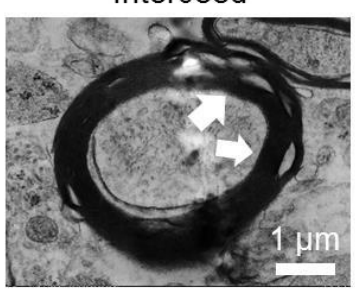

B

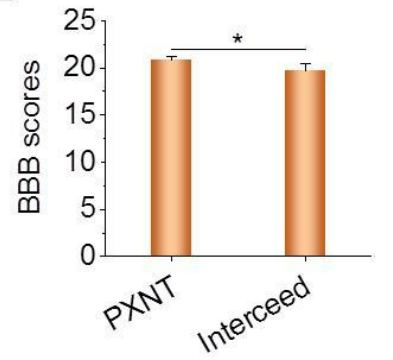

Figure S21. Effects of different barriers on spinal cord morphology and motor function. (A) Representative TEM images of spinal cord morphology at week 3 after treatment with PXNT or Interceed. (B) BBB motor function scores at week 3 post-operation and treatment with either PXNT or Interceed. A score of 21 denotes normal locomotion, and a scale of 0 indicates no movement of the hind limbs. Data are presented as mean \pm SD $(\mathrm{n}=6)$. Statistical significance was assessed by unpaired $t$-test. $* \mathrm{P}<0.05$. 
A

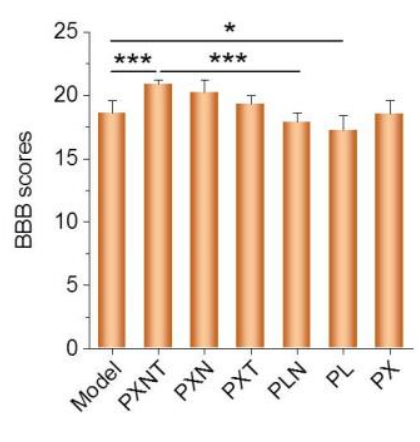

B

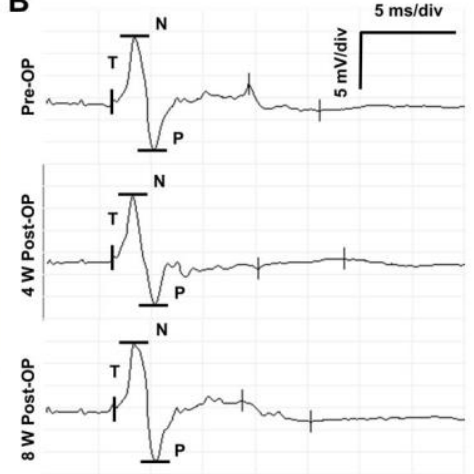

C

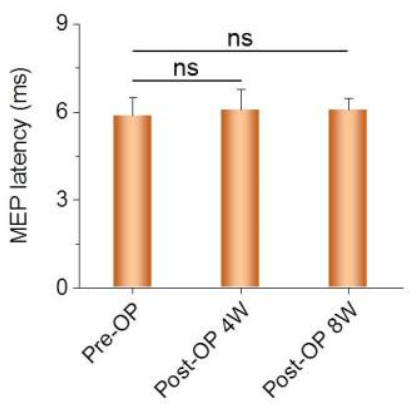

D

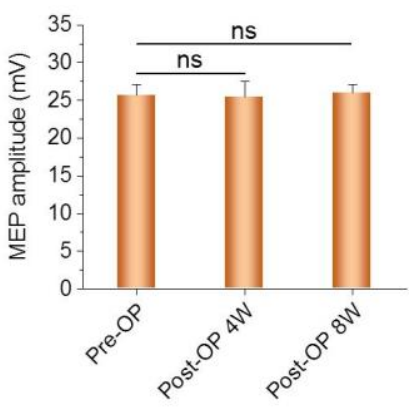

Figure S22. Neurological safety evaluations after different treatments. (A) BBB motor function scores at week 8 post-operation. A score of 21 denotes normal locomotion, and a scale of 0 indicates no movement of the hind limbs. (B) Electrophysiological recordings of motor evoked potentials (MEPs) in rats before (Pre-OP) and post operation (Post-OP) and treatment with PXNT at weeks 4 and 8. (C-D) The quantified data of MEP latency (C) and amplitude (D) at weeks 4 or 8 after treatment with PXNT. Data are presented as mean \pm SD $(A, n=10 ; C$ $\mathrm{D}, \mathrm{n}=6$ ). Statistical significance was assessed by one-way ANOVA tests. ${ }^{*} \mathrm{P}<0.05, * * * \mathrm{P}<0.0005$; $\mathrm{ns}$, no significance. 